This item was submitted to Loughborough's Research Repository by the author.

Items in Figshare are protected by copyright, with all rights reserved, unless otherwise indicated.

\title{
Multi-hazard dependencies can increase or decrease risk
}

PLEASE CITE THE PUBLISHED VERSION

https://doi.org/10.1038/s41558-020-0832-y

\section{PUBLISHER}

Nature Research

VERSION

AM (Accepted Manuscript)

\section{PUBLISHER STATEMENT}

This paper was accepted for publication in the journal Nature Climate Change and the definitive published version is available at https://doi.org/10.1038/s41558-020-0832-y.

\section{LICENCE}

All Rights Reserved

\section{REPOSITORY RECORD}

Hillier, John, Tom Matthews, Robert Wilby, and Conor Murphy. 2020. "Multi-hazard Dependencies Can Increase or Decrease Risk". Loughborough University. https://hdl.handle.net/2134/12489755.v1. 


\section{Multi-hazard dependencies can increase or decrease risk}

2

3 In risk analysis, it is recognised that hazards can often combine to worsen their joint impact, but

4 impact data for a rail network show that hazards can also tend to be mutually exclusive at

$8 \quad{ }^{1}$ John K. Hillier, ${ }^{1}$ Tom Matthews, ${ }^{1}$ Robert L. Wilby, ${ }^{2}$ Conor Murphy

9

$10{ }^{1}$ Geography and Environment, Loughborough University, LE113TU, UK.

$11{ }^{2}$ Irish Climate Analysis and Research UnitS (ICARUS), Department of Geography, Maynooth

12 University, Co Kildare, Ireland.

14 The interplay among natural hazards affects risk globally, and this is expected to evolve as

15 climate changes ${ }^{\text {e.g. 1-3 }}$. Conventional modelling has focused on impacts from each hazard in isolation $^{4,5}$, but this is being transcended by a 'compound event' paradigm for multi-hazards ${ }^{3,6}$.

17 Over meteorological timescales (hours to weeks), hazards like wind and precipitation extremes

18 can combine to exacerbate total risk $^{7-9}$. However, infrastructure operators, government agencies,

19 (re)insurance and health services are also interested in aggregated risk over climatological

20 timescales (seasonal to annual). Using Australia and Great Britain (GB) as examples we

21 illustrate that, from this perspective, some hazards tend to be mutually exclusive due to low-

22 frequency modes of variability. Pairwise views of a multihazard environment that target

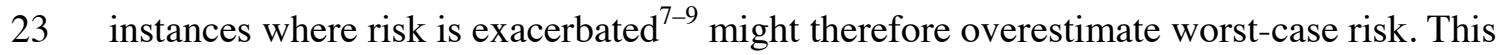

24 complication is one reason for the ongoing development of multi-variate statistical frameworks

25 to better model hazardous extremes ${ }^{10-13}$, but cases are under-reported. Thus, we highlight the

26 need for a broader and more holistic view of multihazard risks, applied at spatial scales meaningful to stakeholders, and including the climatological timescales relevant to them. From 
this standpoint it becomes clear that hazards can be influenced by modes of atmospheric variability in ways that reduce the likelihood of some hazard combinations, thereby moderating tail-end (that is, worst-case) risk (Fig. 1). How such risk moderation works is explained using an analogy of rolling dice, and by relating hazards to climate modes such as the El Niño-Southern

Oscillation (ENSO).

\section{El Niño example}

35 Consider weather-related hazards in Australia. During summer, El Niño tends to reduce the 36 likelihood of tropical cyclones ${ }^{2}$ and flooding ${ }^{8}$, but enhances drought ${ }^{14}$ and wildfire ${ }^{15}$ risk,

37 whereas La Niña drives the opposite conditions. Under the present climate, there are then two regimes or modes of hazard behaviour which are unlikely to co-occur. Concurrent impacts from both cyclone- and drought-related hazards are unlikely when either mode is active, making severe aggregate impacts from all four hazards less likely than might be expected if the two hazard modes were independent. This creates the possibility that, if a tendency for hazard modes to be mutually exclusive exists but is not accounted for, a focus on damaging extremes (individually ${ }^{\text {e.g. } 5,15,16}$ or in pairs/triplets that compound to worsen impacts ${ }^{7-9}$ ) may overestimate risk. Numerous oscillatory climate modes (e.g. ENSO, North Atlantic Oscillation) have been related to the activity level of hazards ${ }^{2,16}$, meaning that cases similar to this Australian example may not be unusual.

\section{Illustration using dice}

49 A probabilistic configuration of multihazards that protects against worst-case combinations can

50 be illustrated by rolling dice. Imagine that flooding and wind damage happen if a six is rolled on

51 a standard die. In traditional risk analysis ${ }^{17}$, flooding and extreme winds are treated as

52 independent phenomena, so would have a die each. A 'worst case' is when both occur (two

53 sixes), on average 1-in-36 throws. If flooding and wind always happen together, however, only

54 one die is needed and both hazards occur if a six is thrown, $1-$ in- 6 rolls. Thus, assessing such 
55 hazards independently underestimates risk ${ }^{7,9,17}$. Flooding and wind damage in a tropical cyclone

are strongly related ${ }^{8}$ and so close to this specific case. To replicate a situation where flooding and

57 wind never happen together, a rule is introduced that rolling a six on one die dictates (somehow)

58 that the other die must score a one; two sixes are not allowed. Hence, neglecting a relationship in

59 which states are mutually exclusive overestimates worst-case risk.

60

61 Where there is a weaker tendency for phenomena to occur together, such as for flooding and

62 wind in extratropical cyclones ${ }^{1,7}$ (Box 1), two similarly loaded dice is a more apt analogy. Both

63 would tend to score high or low such that the chance of two sixes is greater, perhaps 1-in-12. On

64 the other hand, hazards (for example flooding and wildfire in Australia) might tend to be

65 mutually exclusive over a season or year. Then, the loading of the dice would be such that if a

66 six is rolled on the first die the chance of a second six is reduced. This lowers the probability of

67 two sixes, a worst-case impact, to perhaps 1-in-60 throws on average (see Supplementary

68 Discussion 3). These arguments apply to ENSO-driven hazard modes in Australia, and to

69 impactful winter weather in Great Britain.

\section{Great Britain case study}

72 Given that risk relates to potential for loss, an impact-centric approach ${ }^{1,3}$ is essential. The holistic

73 view advocated here requires consideration of multiple hazards and their dependencies at

74 timescales relevant to stakeholders for their hazard-specific exposure (i.e. assets at risk, Fig. 1a).

75 For instance, wintertime weather-related impacts on the rail network in Great Britain (2006-

2018) quantitatively show how such interactions can suppress tail-end risk, mitigating pairwise compound effects (detail in Box 1). In a narrow (bivariate) multi-hazard view, based on the idea

that storms might drive concurrent flooding and wind damage ${ }^{\text {e.g. } 7,8}$, the largest losses for these

79 hazards compound substantially, increasing by $28 \%$ above those simulated assuming that the

80 damage types are independent (Fig. 2d). This view is appropriate for domestic property

81 (re)insurance where these two hazards dominate ${ }^{1}$. However, Network Rail is also exposed to 
82 substantial cold and snow impacts, which themselves compound by $+8 \%$ during winter.

83 Assuming now that losses are paired, but the pairs are independent given that no published study explicitly suggests otherwise, yields a simulated overall increase of $+17 \%$. Figure $2 \mathrm{~d}$ demonstrates that these calculations would overestimate risk as the total effect of all dependencies acting together on these four hazards results in little net effect (approx. -1\%). This occurs because when flooding and wind incidents are prevalent, impacts due to cold and snow are muted. Therefore, a broad multi-hazard approach evidently leads to a very different conclusion from bivariate view(s) (Fig. 1b).

90

Mutual exclusivity is not self-evident. Storm Ciara $\left(8-10^{\text {th }}\right.$ February 2020) led to disruption from snow, flooding, and wind damage. However, some hazards are more likely under particular synoptic-scale conditions ${ }^{\text {e.g. } 16,18,19}$ (weather types ${ }^{20}$ ), so if the frequency of a weather type is high over the season the damage from its associated hazards will also be high. Mutual exclusivity arises as the frequency of different weather types trade off against one another ${ }^{20}$. The greater the difference in hazard likelihood between weather types, and the clearer the seasonal trade-off in their frequency, the stronger this effect will be.

For GB, wintertime frequencies of cyclonic (flood and wind damage ${ }^{7,18}$ ) and anticyclonic/northeasterly (cold and snow damage ${ }^{5,19}$ ) weather types show strong compensation ${ }^{20}$ explaining the emergence of 'hazard modes' described in Box 1. These modes, however, may not persist in a future climate. Even if the trade-off in circulation types persists, air masses associated with cold and snow hazards may warm sufficiently to no longer yield damage, reducing annual average losses by $\sim 30 \%$ (see Fig. 2a). To resolve the effect of such dependencies, and to test simplified are needed. 
109 In summary, dependencies between causative hazards can both compound and suppress risk e.g.

$111^{1,3,9}$ (Fig 1b, 2d). Both over and under-estimation of risk is problematic for the systems and

111 people affected. To neither waste resources nor to be underprepared, scientists, society and

112 decision-makers need to be alert to both. To date, however, the focus of the modelling

113 community (especially within climate science) has been on those aspects of dependency that

114 make outcomes worse. Our contribution highlights that the opposite can also be true (Fig. 1b).

115 We therefore advocate a broad view of compound risk - a refinement of the established

116 'compound event' viewpoint that might better serve hazard and risk management communities.

118 The term 'compound', as applied to risks, developed from its meaning 'make (something bad)

119 worse; intensify the negative aspects of ${ }^{3,6,17}$ (see Supplementary Discussion 2). Recently, the

120 definition has been expanded ${ }^{3,6}$, but only rarely is a beneficial side-effect ${ }^{3}$ or reduction of risk

121 noted as a caveat ${ }^{21}$, and the importance of these tends to be downplayed. We believe that this

122 wider description, including such positive connotations, is viable as the word compound also

123 means to 'mix or combine constituents', in this case a mix of hazards. An 'event', however, is an

124 occurrence rooted in a specific time and place. Thus, perhaps the phrase 'compound event risk'

125 could be explicitly kept narrow. Conversely, we advocate that 'compound risk' be applied more

126 holistically to accommodate both compound events ${ }^{3,6}$ that increase impact severity as well as

127 those impacts that combine within extended timeframes and combine in ways that are mutually

128 exclusive.

130 Based on the examples above, situations are likely common globally where multihazard risk is

131 systematically suppressed with respect to selected narrow (e.g. bi-variate or pairwise)

132 configurations. We suspect that these are under-diagnosed due to (i) lack of systematic collection

133 and analysis of impact data, (ii) difficulties in comparing hazards (for example, time-lags

134 between extreme events, different hazard metrics ${ }^{1,17}$, time-lags between end-member conditions 
135 like El Niño/La Niña), and lastly (iii) a need for greater awareness that such situations should be

136 identified. The subtle balance between hazards is also non-stationary as climate changes ${ }^{\text {e.g. } 2,3}$, so

137 diagnosis is needed for future climates. Moreover, dependencies are not only between causative

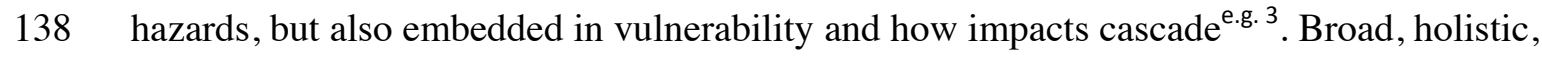

139 stakeholder relevant assessments of compound risk for multihazard environments are therefore

140 needed to improve assessments of current and future risk.

Fig. 1: Illustration of plausible effects when the activity of hazard(s) switches between climatologically controlled modes of behaviour, based on Great Britain. a) Impact-centric ${ }^{1,3}$

149 conceptualisation of the multihazard system. Two hazard modes, each associated with a

150 dominant wind direction (blue arrows), drive six hazards (circles). Rail infrastructure (red) is

151 exposed to all six hazards, whilst (re)insurance (black) is primarily concerned with only two in

152 Mode 2. b) Losses in terms of magnitude and frequency, with rare 'worst cases' on the righthand

153 side (grey band). A conventional view that does not consider dependencies (grey) might

154 underestimate risk if two hazards (for example, flood and wind) compound. However, where

155 exposed assets are subject to hazards driven by two opposing modes (red) compounding effects

156 are suppressed, so care is needed to avoid overestimating risk. Solid arrows represent effect

157 magnitudes seen within the Network Rail loss data (Fig. 2, Box 1), with dashed lines indicating 158 plausible stronger effects. 


\section{Box 1: A case study of compound risk in Great Britain}

160

161 A case study of weather-related impacts (Fig. 2a, Supplementary Discussion 1) on the rail

162 network in Great Britain (GB) illustrates that interactions may suppress tail-end (i.e. worst) risk,

163 mitigating pairwise compound effects (Fig. 2d). Here, impact is expressed as delays to trains

164 which, when monetized, costs Network Rail an estimated £64.6 million annually. Most (72.5\%)

165 of these costs occur in the 'winter' half-year (Oct-Mar). Thus, winter is selected as our temporal

166 domain for a seasonal-scale analysis, which in GB is hydro-meteorologically distinct from

167 summer (see Supplementary Figure 1).

168

169 Fig. 2d shows that from a narrow (bivariate) multihazard perspective, the largest losses (i.e. 13

170 year return period) for flooding (Fl) and wind damage (Wi) compound substantially (+28\%).

171 Simulation modelling ${ }^{1,10}$ is conducted to demonstrate that this observation (dark blue line) is not

172 due to chance $(\mathrm{p}<0.05)$ by breaking all hazard inter-relationships. In the simulation (see

173 Supplementary Discussion 1), this was achieved by shuffling the years in which losses occur

174 independently for each hazard, and 10,000 of these random realizations are used (dashed line).

175

176 However, a wider (multivariate) view yields a distinctly different outcome. When one set of

177 hazards in GB is active, the other main group is typically not. Fig. 2b shows that the categories

178 of loss may be, visually or otherwise, grouped into sub-sets that tend to coincide (that is, are

179 correlated). Specifically, when Fl and Wi incidents are prevalent, impacts due to cold (Co) and

180 snow (Sn) are muted. When summed in pairs (e.g. Sn+Co vs Fl+Wi), there is no overlap

181 between the years in which each pair's top five losses occur ( $p=0.024, \chi^{2}$ test), and an inverse

182 correlation is evident (Fig. 2e). In Fig. 2d, this mutual avoidance expresses itself as the largest

183 losses (i.e. 13 year return period) being suppressed to the level of entirely random selection

184 (dashed line) even as each of the pairs is demonstrated to compound (blue lines). This new

185 observation adds to growing evidence of a seasonal scale Fl-Wi association for $\mathrm{GB}^{1,7,18}$. 
187 We propose the term 'hazard modes' for sub-sets of partially associated hazards (Fig. 2b). For

188 GB, we hypothesize a simple westerly vs north-easterly driver of the hazard modes (Wi + Fl:

189 westerly, or Sn + Co: north-easterly: Fig. 2c). Low pressure systems (named storms) are

190 associated with westerly and cyclonic synoptic-scale atmospheric patterns ${ }^{18}$, and although

191 individual storms are typically viewed as either causing Fl or Wi damage ${ }^{4,16}$ some cause both

192 (e.g. Storm Desmond); particular seasons have also been identified as notably 'wet and

193 stormy ${ }^{, 18}$. Opposing this behaviour, episodes of extreme wintertime cold or snow in GB are

194 typified by advection of cold air from the north and east, and 'blocking' anticyclonic

195 circulation $^{\text {e.g. 5,19. }}$. The wintertime frequency of mobile westerly and cyclonic flows are known to

196 trade off against more stable anticyclonic conditions ${ }^{20}$. Here we show that the North Atlantic

197 Oscillation is a useful metric to capture these behaviours through its correlations with the hazard 198 modes (Fig. 2f,g).

200 Fig. 2: Winter multihazard impacts in Great Britain. a) National total winter (October-

201 March) losses for nine hazards affecting Network Rail, of which seven are substantive, i.e.

$202<0.1 \%$ of total losses. b) Correlations between the loss classifications; stars denote statistical significance; $*<0.05$ (i.e. $95 \%$ confidence), $* *<0.01, * * *<0.001$. c) Illustration of the proposed 'hazard modes'. d) The effect of interdependencies between hazards upon seasonal loss totals, calculated as the difference between observations (solid lines) and simulation modelling in which randomisation has been used to break any process-based linkages between hazards (dashed line). Larger, rarer losses have greater return periods in this exceedance probability plot.

Loss values greater than zero indicate that selected hazard combinations compound, or alternatively are suppressed. Statistical significance of the tendency to compound is computed using the AEP method ${ }^{1}$ and simulation. Details of data and simulation methods are provided in

211 Supplementary Information. e)-g) Correlations between losses, and with the North Atlantic

212 Oscillation (NAO). Pearson's $r$, grey shading is 95\% confidence interval for the trendline. 


\section{References}

215 1. Hillier, J. K., Macdonald, N., Leckebusch, G. C. \& Stavrinides, A. Interactions between

216 apparently primary weather-driven hazards and their cost. Env. Res. Lett. 10, 104003 (2015).

217 2. Steptoe, H., Jones, S. E. O. \& Fox, H. Correlations between extreme atmospheric hazards and

218 global teleconnections: Implications for multi-hazard resilience. Reviews of Geophysics 55,

$219 \quad 50-78(2017)$.

220

3. Zscheischler, J. et al. Future climate risk from compound events. Nature Climate Change (2018) doi:10.1038/s41558-018-0156-3.

4. Lavers, D. A. et al. Future changes in atmospheric rivers and their implications for winter flooding in Britain. Environmental Research Letters 8, 034010 (2013).

5. Bieli, M., Pfahl, S. \& Wernli, H. A Lagrangian investigation of hot and cold temperature extremes in Europe. Quaterly Journal of the Royal Meteorological Society 141, 98-108 (2015).

6. Leonard, M. et al. A compound event framework for understanding extreme events. WIREs Climate Change 5, 113-128 (2014).

7. De Luca, P., Hillier, J. K., Wilby, R. L., Quinn, N. W. \& Harrigan, S. Extreme multi-basin flooding $230 \quad$ linked with extra-tropical cyclones. Env. Res. Lett. 12, 114009 (2017).

8. Khouakhi, A. \& Villarini, G. Contribution of tropical cyclones to rainfall at global scale. Journal of Climate 30, 359-372 (2017).

9. Wahl, T., Jain, S., Bender, J., Meyers, S. D. \& Luther, M. E. Increasing risk of compound flooding from storm surge and rainfall for major US cities. Nature Climate Change 5, 10931097 (2015).

236 10. Wyncoll, D. \& Gouldby, B. Integrating a multivariate extreme value method within a 237 system flood risk analysis. Journal of Flood Risk Management 8, 145-160 (2013). 
11. Serinaldi, F. \& Papalexiou, S. M. Random fields simplified: Preserving marginal distributions, correlations, and intermittency, with applications from rainfall to humidity. Water Resour. Res. 56, e2019WR026331 (2020).

12. Wadsworth, J. L., Tawn, J. A., Davidson, A. C. \& Elton, D. M. Modelling across extremal dependence classes. Journal Of The Royal Statistical Society Series B 79, 149-175 (2017).

13. Bevacqua, E., Maraun, D., Haff, H. I., Widmann, M. \& Vrac, M. Multivariate statistical modelling of compound events via pair-copula constructions: analysis of floods in Ravenna (Italy). Hydrol. Earth Syst. Sci. 21, 2701-2723 (2017).

14. Nicholls, N. The El Niño / Southern Oscillation and Australian Vegetation. Vegetatio 91, 23-36 (1991).

15. Mariani, M., Fletcher, M. S., Holz, A. \& Nyman, P. ENSO controls interannual fire activity in southeast Australia. Geophys. Res. Lett. 43, 10891-10900 (2016).

16. Walz, M. A., Donat, M. G. \& Leckebusch, G. C. Large-Scale Drivers and Seasonal Predictability of Extreme Wind Speeds Over the North Atlantic and Europe. Journal of Geophysical Research: Atmospheres 123, 11518-11535 (2018).

17. Kappes, M. S., Keiler, M., von Elverfeldt, K. \& Glade, T. Challenges of analyzing mulithazard risk: a review. Nat. Hazards 64, 1925-1958 (2012).

18. Matthews, T., Murphy, C., Wilby, R. L. \& Harrigan, S. Stormiest winter on record for Ireland and UK. Nature Climate Change 4, 738-740 (2014).

19. Mayes, J. Regional weather and climates of the British Isles - Part 2: South East England and East Anglia. Weather 68, 59-65 (2013).

20. Briffa, K. R., Jones, P. D. \& Kelly, P. M. Principal component analysis of the Lamb

260 catalogue of daily weather types: Part 2, Seasonal frequencies and update to 1987. International Journal of Climatology 10, 549-563 (1990). 
262 21. Gill, M. \& Malamud, B. D. Reviewing and visualizing the interactions of natural hazards.

263 Reviews of Geophysics 52, 680-722 (2014).

264 
a)

M.tim

(Re)insurance

?

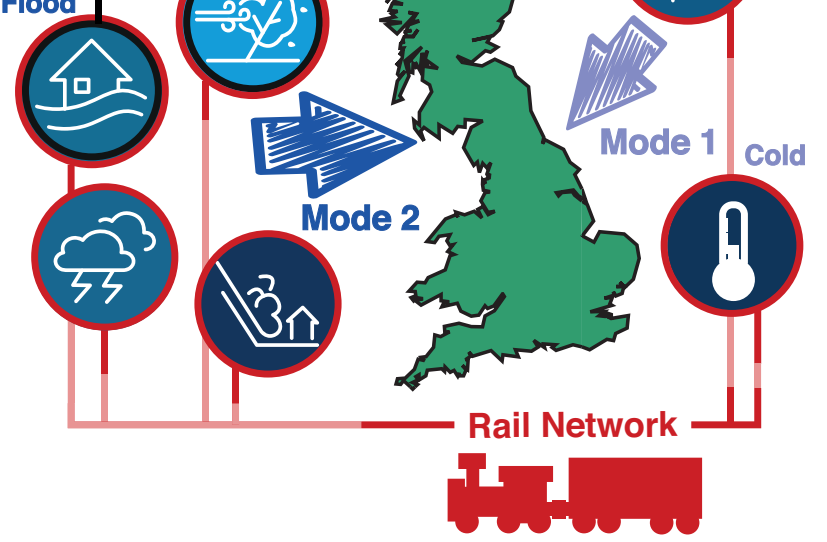

b)

$A$ 

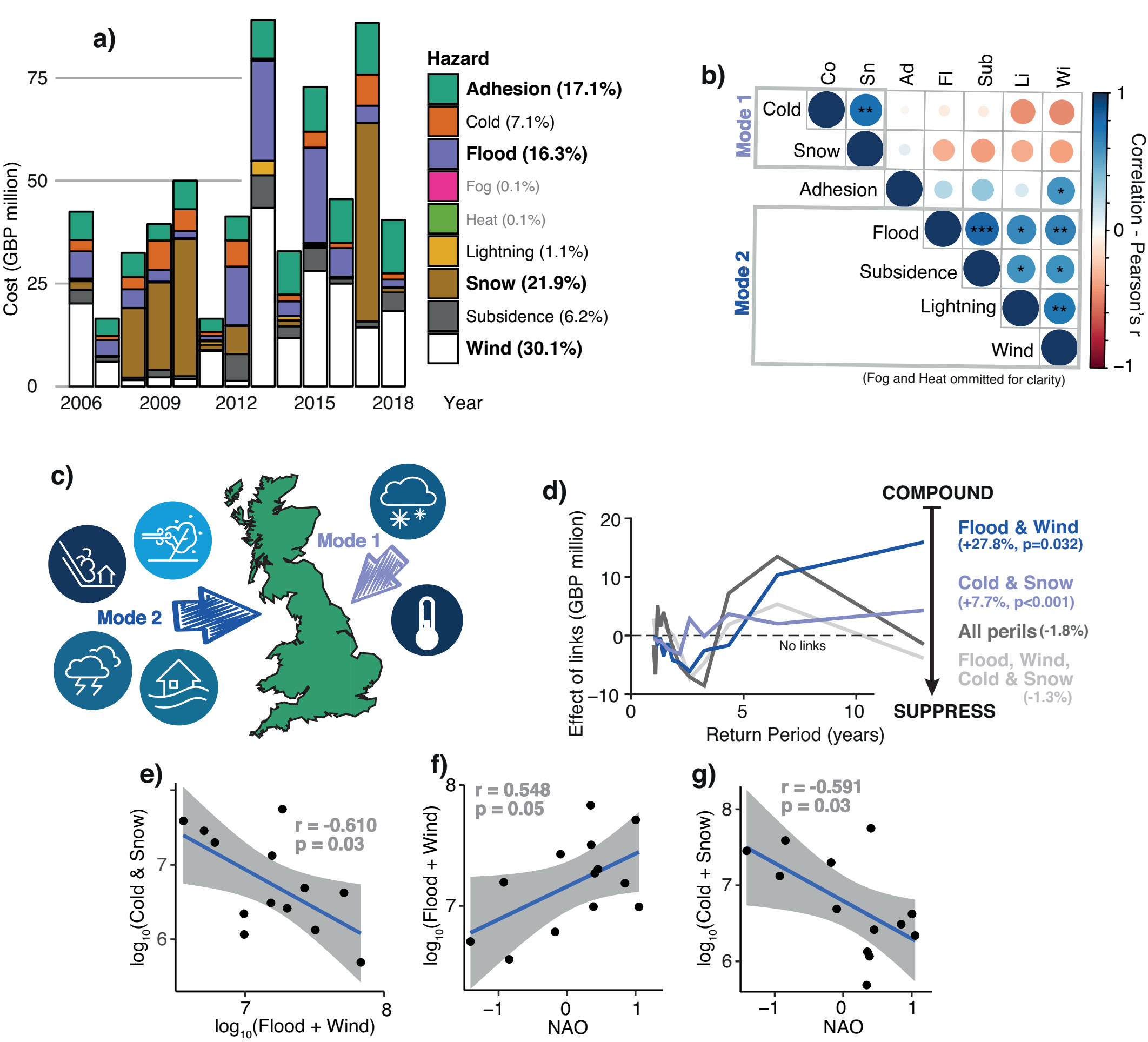


\section{Multi-hazard dependencies can increase and decrease risk}

${ }^{1}$ John K. Hillier, ${ }^{1}$ Tom Matthews, ${ }^{1}$ Robert L. Wilby, ${ }^{2}$ Conor Murphy

${ }^{1}$ Geography and Environment, Loughborough University, LE11 3TU, UK.

${ }^{2}$ Irish Climate Analysis and Research UnitS (ICARUS), Department of Geography, Maynooth University, Co Kildare, Ireland.

\section{Supplementary Information}

This document contains supplementary material. There are three main sections. First Supplementary Discussion 1 (S1), relates to the case study of weather-related impacts on the rail network in Great Britain. This expands upon the study contained in main text and Box 1. Within this, sub-sections on data and methods are referred to so that the reader can go into this level of detail if desired. Second, in S2, the widening scope of term 'compound' as applied to risks is more fully documented. Third, in S3, dice games using only standard dice are set out to illustrate the impacts of dependencies, including partial dependency.

\section{Supplementary Discussion 1. Case Study: Compound risk in Great Britain}

In the main text and Box 1, a case study of weather-related impacts on the rail network in Great Britain (GB) illustrates that interactions may suppress tail-end (i.e. worst) risk, mitigating pairwise compound effects. The data used in the study are detailed in section S1.1 below, and a formal presentation of the simulation methodology ${ }^{1}$ is in S1.2 'Methods'. 


\section{S1.1 Data}

Weather Related Incident Impact Data (WRIID) is Schedule 8 delay data, manually attributed to nine hazards by Network Rail (NR), who are the owner and infrastructure provider of most of the rail network in GB. WRIID contains 182,000 incidents (2006-2019) that have each been assigned to a hazard and impact location (i.e. the originating site of delays). These data have been used previously for work on heat ${ }^{2,3}$, but not for multi-hazard analysis. Impact is expressed as delays to trains which, when monetized, costs Network Rail an estimated $£ 64.6$ million annually. Most (72.5\%) of these costs occur in the 'winter' half-year (Oct-Mar) ${ }^{4}$. Thus, winter is selected as our temporal domain (i.e. seasonal scale), which in GB is hydro-meteorologically distinct from summere.g. 5 (see Supplementary Figure 1).

NR's planning year is April-March, i.e. Q1/Q2 is 'Summer' and Q3/Q4 'Winter' (October-March). The year-end is aligned with the UK hydrological/meteorological year ${ }^{4,6}$ as defined by the National River Flow Archive - https://nrfa.ceh.ac.uk/. Thus, assignments in Fig. 2 are for the year in which the winter initiates i.e. 2017 for Oct 2017 to Mar 2108.

Values $L_{i j}$ are winter totals, disaggregated by year $i$ and hazard $j$. For the time span 2006-2018 there are 13 winters, i.e. $y=13$ and $i=\{1,2 \ldots . y\}$. There 9 hazards, i.e. $h=9$ and $j=\{1,2 \ldots . h\}$, giving $n=117$ data points. These alone are used in the analysis (i.e. Figs $2 b, d$ ). Thus, all data in this study are published in Fig. 2a.

In other loss datasets, mis-categorisation of some losses is a recognized issue. For instance, in the Association of British Insurers (ABI) 'General Insurance Statistics ${ }^{17}$, some pluvial inundation (surface water flooding) is known by practitioners to be reported as wind damage because it is 
associated with stormy weather. This reporting artefact makes it difficult to discern a physically driven component of any correlation between flooding $(\mathrm{FI})$ and wind $(\mathrm{Wi})^{1}$. Such an artefact does not appear to be present in WRIID. In winter FI-Wi are dependent $(r=0.68, p<0.01$, Fig. 2b), but in summer there is no demonstrable relationship ( $r=-0.05$, Supplementary Figure 1$)$, and it is difficult to imagine how a reporting artefact might exist in winter and yet be avoided in summer. Other relationships also change (Supplementary Figure 1), suggesting that this inference applies to the other hazards. The summer multi-hazard system, however, is not the subject of this paper.

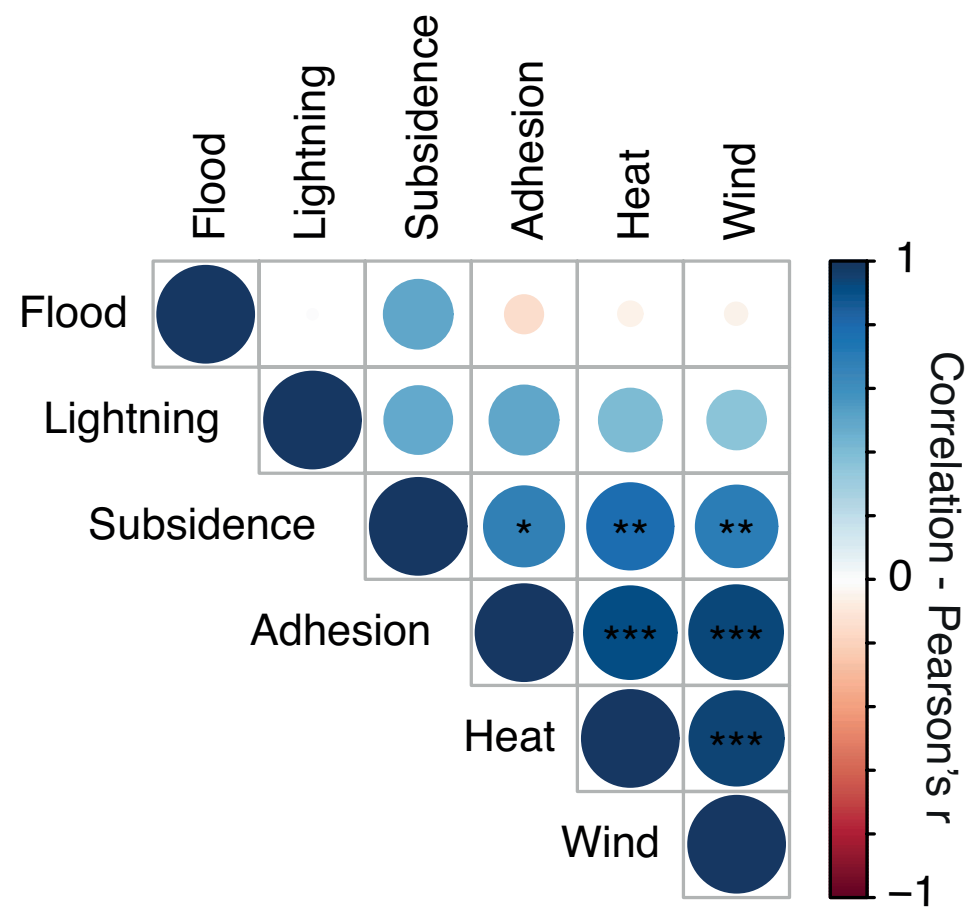

Supplementary Figure 1: Summertime multi-hazard impacts in Great Britain. Correlations between the loss classifications; stars denote statistical significance - * $<0.05$ (i.e. $95 \%$ confidence), $* *<0.01, * * *<0.001$. Cold, snow and fog losses are negligible in the summer. 


\section{S1.2 Methods}

Academic literature describing approaches to multi-hazard interactions is limited, mainly applied to combinations of a pair of hazards, and interdependencies between receptors (such as people and infrastructure) are rarely explicitly modelled e.g. 8 .

The method used here combines recently advocated methods. It is designed to be a pragmatic yet powerful approach for gaining insights into compound risk in highly multi-hazard systems (i.e. $\geq 3$ extremes), which works even with multiple time-lagged events, varied hazard types and data time-series of limited length. Key elements of the method are using (i) loss data ${ }^{1,9,10}$, (ii) annual/seasonal time-scales for hazard correlation ${ }^{1,9}$ and (iii) simulation modelling of Aggregate Exceedance Probability (AEP) ${ }^{1,11}$. These are described below sequentially, along with an explanation of how the correlation matrix is constructed.

Use of loss data: Using loss data is an approach that was recently categorised as 'bottom-up' and strongly advocated ${ }^{12}$. Quantitative, impact-centric risk analyses based on observed losses ${ }^{1,9,10}$ or even proxies ${ }^{13}$ are sparse, but the use of losses permits direct comparisons between hazards overcoming a key analytical difficulty encountered by previous multi-hazard analyses (e.g. time-lags between events, different hazard metrics etc. $)^{14}$.

Use of a seasonal/annual time-frame: Extended (up to annual) timeframes have been clearly included in major reviews of multi-hazard risk ${ }^{14,15}$, and seasonal scales for storm prediction (e.g. Tropical Cyclones) have been extensively used ${ }^{16-19}$, but quantitative analysis of multi-hazard risk on a seasonal scale are relatively rare e.g. 1,9,20. Typically, compound events involve individual episodes set within a relatively limited timeframe (e.g. $<14$ days $)^{21,22}$. One advantage of using 
numerous events that occur across a season is in mitigating the aleatory uncertainty (i.e. random noise) in particular events (e.g. impact might depend on whether it is night or day, or the exact time distribution of rain), with these cancelling out with a sum or mean to give a better estimate of tendency with respect to a driver (e.g. climate mode) via the central limit theorem. A second advantage is that antecedence (such as water saturation of the ground) is accounted for, which is important in hazards like flooding ${ }^{23}$.

Correlation matrix: Relationships between hazards are expressed (Fig. 2b) in terms of Pearson's correlation coefficient $(r)$, despite known limitations ${ }^{24}$. Pearson's $r$ is selected for familiarity, simplicity and because the non-parametric calculations in this work regarding Aggregate Exceedance Probability (AEP) curves in Fig. 2d (see 'Simulation modelling' below) account for the net effect of both the dependency structure and marginal distribution of the data ${ }^{25,26}$ (Fig. 2a), which $r$ reflects.

For completeness, Supplementary Figure 2a isolates the dependency structure using Spearman's rank correlation ${ }^{24,25}$, and Supplementary Figure $2 \mathrm{~b}$ uses logarithms of the losses to correct for the marginal distribution although this relies on assuming the type of that distribution ${ }^{27}$, in this case selecting the log-Normal loss distribution ${ }^{28}$. The proposed hazard modes and clustering structure (i.e. determination of which hazards are most closely related) remain very similar to Fig. $2 \mathrm{~b}$ even if statistical significances are mainly estimated as lower (e.g. within Mode 2). The other main difference is that the inverse relationship between the two modes is emphasized more strongly (e.g. for Snow and Wind).

R functions rcorr(), corrplott and hclust() $)^{29}$ are used. 
a)

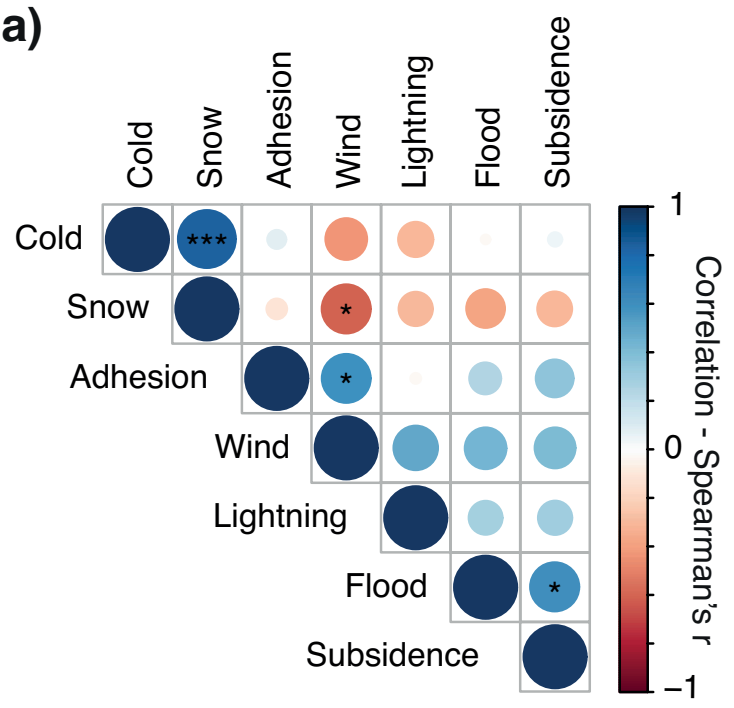

b)

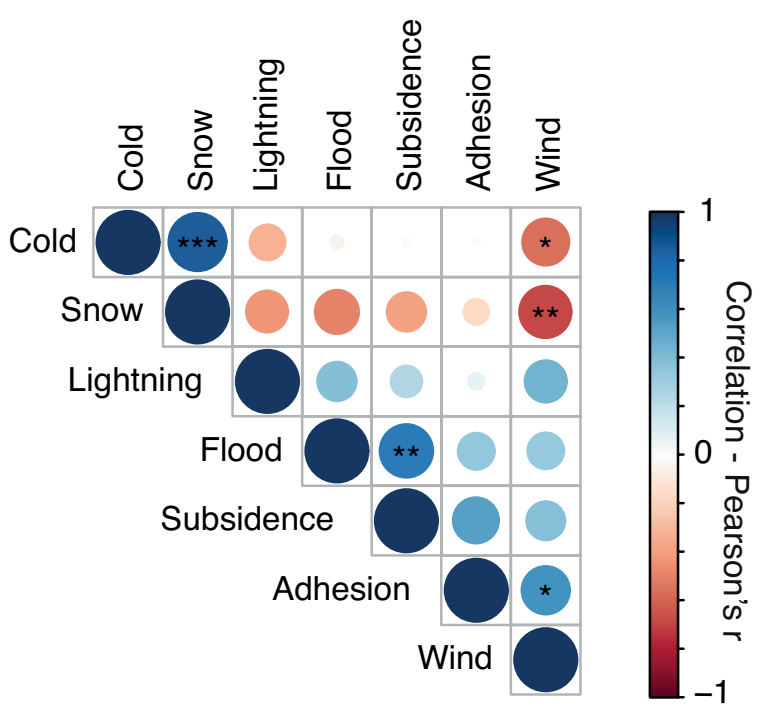

\section{Supplementary Figure 2: Winter dependency structure between the loss classifications. a)}

Spearman's rank, which is a less powerful test but isolates the structure from the marginal distribution of losses. b) Pearson's $r$ for logarithms of losses. Stars denote statistical significance $-*<0.05$ (i.e. $95 \%$ confidence), $* *<0.01, * * *<0.001$. The ordering of the hazards is objectively determined by the clustering algorithm used (see text).

Simulation modelling: The co-occurrence of extremes has been assessed using a null hypothesis of independence ${ }^{21,30}$ as has the magnitude of a hazard subject to multivariate drivers ${ }^{11}$. In considering compound risk ${ }^{15}$ however, it is of fundamental importance to ascertain the difference in impact (e.g. financial cost) between realizations of reality where dependencies exist and realizations where they do not. But, this is rarely done ${ }^{1,8}$. We, therefore, use the one methodology to establish correlation on this basis - the AEP method ${ }^{1}$. AEP is exceedance probability (EP) for aggregate losses, which is directly applicable to the WRIID dataset in Fig. 2a as $L_{i j}$ are totals from numerous events over a season. The AEP method has six stages: 
(1) Select the sub-set of at least two hazards of interest e.g. $h_{\text {sub }}=\{1,2,8\}$, which may be all the hazards, and compute total seasonal observed (i.e. aggregate) losses $A L_{i}=\sum_{j} L_{i j}$ where $j \in$ $h_{\text {sub }}$.

(2) Calculate empirical (i.e. observed) AEP values for $A L_{i}$, which implicitly contains all interdependencies within the system, albeit potentially masked by unrelated perturbations or 'noise'. These are computed by the standard textbook method ${ }^{31}$. Specifically, sort $A L_{i}$ in order of descending magnitude, assigning each an index (i.e. row) in the list $k . A L_{k}$ is then the $k^{\text {th }} A L_{i}$ value in the list. As each year occurs once the probability $p$ of each is $1 / y$, the observed exceedance probability $E P_{k}$ is $k / y$, and the associated return period (e.g. Fig. $1 d$ ) is $y / k$.

(3) Compute the gradient $g_{\text {obs }}$ of exceedance probability (i.e. $E P_{k}$ ) plotted against observed losses (i.e. $A L_{k}$ ). Specifically, a linear model is fitted by ordinary least squares to estimate coefficients in $A L=g^{*} E P+c$. Thus, $g_{\text {obs }}$ is a single value quantifying correlation in losses resulting from all interactions at their empirically observed magnitudes.

(4) Conduct 10,000 simulations $m=\{1,2 \ldots .10,000\}$ wherein randomisation is used to eradicate any possible dependencies between hazards. In each simulation, for each hazard $j$ separately, losses $L_{i j}$ are randomly re-assigned to a year and denoted $L_{i j m}$. Then, steps (1) to (3) are repeated for each simulation to generate $A L_{i m}, A L_{k m}$ and $g_{m}$.

(5) Calculate the mean simulated AEP curve with dependencies eliminated, i.e. $\overline{A L_{k m}}=$ $\frac{1}{m} \sum_{m} A L_{k m} . \overline{A L_{k m}}$ vs $E P_{k}$ can then be compared to the observed AEP curve. Visually this is most effectively performed by plotting the difference between the observed and mean simulated curves (Fig. 2d). 
(6) Establish whether or not the observed compounding effect within the subset of hazards could have occurred by chance. The null hypothesis is that there is no compounding effect i.e. $\mathrm{H}_{0}: g=0$, with an alternative hypothesis that a compounding effect exists i.e. $\mathrm{H}_{1}: g<0$; $x$-axis is inverted by use of probability, not the return period. By the central limit theorem the distribution of $g$ is roughly normal, but this test is non-parametric as the simulation-based $p$ value of $g_{\text {obs }}$ is calculated directly. Illustratively, if $g$ for 24 realizations $i$ is smaller than $g_{\text {obs }} p=$ 0.0024 and the null hypothesis that $g_{\text {obs }}=0$ (i.e. no effect of dependencies) can be rejected. Note that this simulation requires no assumptions about statistical distributions, dependency structure, physical processes, or climate modes.

\section{Supplementary Discussion 2. Defining Compound: Widening scope of term 'compound' as} applied to risks

In the Oxford English Dictionary (OED), the word 'compound' has several meanings. When used as a verb, these include to 'mix or combine (ingredients or constituents)' and to 'make (something bad) worse; intensify the negative aspects of '. It is conceivable that both of these concepts apply when 'compound' is applied in the context of risk $12,14,15,32,33$.

In 1971 Hewitt ${ }^{32}$ characterized a compound hazard as 'several elements acting together above their respective damage threshold'. In 2010 Kappes $^{14}$ distinguished two types of hazard relationship: (1) where one hazard is triggered by another and (2) where the disposition (i.e. magnitude or frequency) of one hazard is altered by another either simultaneously or in a following season. Thus, a typology is formed for 'acting' and the timeframe of 'together' is expanded. By 2012, the IPCC definition ${ }^{33}$ was extended to include (i) the confluence of multiple 
sub-extreme conditions 'combinations of events that are not themselves extremes but lead to an extreme event or impact when combined', and (ii) when impact is amplified by the extremes (e.g. temperature and wind) combining with underlying conditions (e.g. fuel for a wildfire). However, this detail introduces ambiguities, leading Leonard in $2014^{15}$ to propose a more general definition i.e.

'A compound event is an extreme impact that depends upon multiple statistical variables or events'

Here, the emphasis on impact is useful, but the importance of longer timeframes involved is downplayed by use of the word 'event' rather than 'risk'. In 2018, Zscheischler ${ }^{12}$ emphasizes that '[ignoring] dependencies can lead to the underestimation of risk', before defining compound weather/climate events as:

'The combination of multiple drivers and/or hazards that contributes to societal or environmental risk'

This maintains many of the benefits of Leonard's definition and usefully distinguishes drivers (i.e. underlying conditions such as hyrdo-meteorological extremes), from hazards (i.e. the physical property causing damage such as water depth) and from risk (i.e. impact and its likelihood). It explicitly generalizes previous definitions and continues the expansion of the scope of the term. Rarely, a positive side-effect ${ }^{12}$ or reduction of risk is noted as a caveat ${ }^{34}$, but the importance of these is downplayed. However, there are combinations of drivers and/or hazards that contribute to risk (i.e. losses in Figs. 1, 2) in which the combinations moderate (i.e. suppress) rather than worsen (i.e. compound) risk. Thus, ambiguity is introduced into the term 
'compound event hazard/risk'. Either, the definition should be narrowed, or explicit attention is needed to clarify that moderation/mitigation is included within this (or a similar) term such as 'compound hazard/risk', within which an emphasis on the second OED definition (i.e. worsen) is not obligatory.

\section{Supplementary Discussion 3. Dice Games}

A series of exercises or 'games', using only standard dice, provide a way to verify our statistical assertions about how full and partial dependency between two hazards affect the likelihood of a 'worst case' where both occur. These directly relate to the examples in the main text, with the difference that an 'event' (e.g. a flood) occurs if the score on the relevant die is defined as 'high' rather than being a six only. The independent case in Game 1 is the baseline to which the other scenarios are compared. All Games are set so that the overall level or hazard (i.e. events of either type occurring) is constant, set at 1-in-3 times that a die is rolled.

(1) Independent hazards: Imagine that flooding and wind damage happen if a 'high' value is rolled on a standard die. In traditional risk analysis ${ }^{14}$, flooding and wind are viewed as independent phenomena that have a die each, and a 'worst case' occurs when both happen (i.e. two high values). Define high as when a 5 or 6 is rolled. Then, a worst case happens 1-in-9 times $\left(\frac{1}{3} \times \frac{1}{3}=\frac{1}{9}\right)$. Roll both dice together a number of times to verify this. Also, verify that overall hazard is set so that a high value occurs 1-in-3 times a die is rolled (i.e. taken individually, count all rolls and all high values).

(2) Completely positively dependent hazards: If flooding and wind always happen together, only 1 die is needed to represent both of them. Define high as when a 5 or 6 is rolled. Verify 
that both hazards occur 1-in-3 throws. Verify that, as in Game 1, overall hazard is set so that a high value occurs 1-in-3 times.

(3) Completely negatively dependent hazards: If flooding and wind never happen together, two high values are simply not allowed. No dice are really needed to determine that the chance of a worst case (i.e. both together) is zero however, to complete this analogy, define high as when a 5 or 6 is rolled, and low is when a 1 or 2 is rolled. Roll one die then the other. If both dice are high, swop the second to be equivalently low. If both dice are low, swop the second to be equivalently high. Sixes are swopped to be ones, and visa versa, and fives and swopped with twos. After this manipulation, verify that both hazards never occur together. Verify that, as in Game 1, overall hazard is set so that a high value occurs 1-in-3 times.

(4) Partial positive dependency: Where there is a weaker relationship between phenomena, such as for flooding and wind in extratropical cyclones, two loaded dice are a more apt analogy. This can be replicated with standard dice if they are thrown sequentially so that the second 'knows' what the first has done. 'High' on the first is defined as 5 or 6 , and 'low' as 1 or 2 . On the second, high is more likely (i.e. 4,5,6) after a high on the first die, less likely (i.e. 6 only) when the first is low, and unaltered for intermediate values. Verify that both hazards occur 1in-6 times $\left(\frac{1}{3} \times \frac{1}{2}=\frac{1}{6}\right)$, more than independence (Game 1) yet less than complete dependence (Game 2). Verify that, as in Game 1, overall hazard is still set so that a high value for an individual hazard occurs 1-in-3 times a die is rolled. If desired, a hazard can be randomly allocated to a die before each pair are thrown in sequence (e.g. by flipping a coin), but this does not alter the outcome.

(5) Partial negative dependency: if hazards (e.g. flooding and wildfire) tend to be mutually exclusive within a day, season or year, depending upon the spatial domain considered (e.g. Australia), imagine two dice loaded to reflect this. This loading can be replicated with standard dice if 'high' on one is defined as 6 only, and on the other as 4,5 or 6; decide which before 
rolling - dice of different colours may be useful here. Roll both dice together. Verify that both hazards occur 1-in-12 times $\left(\frac{1}{6} \times \frac{3}{6}=\frac{1}{12}\right)$, more than complete mutual exclusivity (Game 3 ) yet less than independence (Game 1). Verify that, as in Game 1, overall hazard is still set so that a high value for an individual hazard occurs 1-in-3 times a die is rolled. If desired, a hazard can be randomly allocated to a die before each pair are thrown (e.g. by flipping a coin), but this does not alter the outcome.

\section{References}

1. Hillier, J. K., Macdonald, N., Leckebusch, G. C. \& Stavrinides, A. Interactions between apparently primary weather-driven hazards and their cost. Env. Res. Lett. 10, 104003 (2015).

2. Ferranti, E. J. S. et al. Heat-related failures on South-East England's railway network: insights and implications for heat-risk management. Weather Climate and Society 8, 177-191 (2016).

3. Ferranti, E. J. S. et al. The hottest day on the railway network; insights and thoughts for the future. Meteorological Applications 5, 195-208 (2018).

4. Lavers, D. A. et al. Winter floods in Britain are connected to atmospheric rivers. Geophys. Res. Lett. 38, L23803 (2011).

5. Svensson, C. et al. Long-range forecasts of UK winter hydrology. Env. Res. Lett. 10, 064006 (2015).

6. Wilby, R. L. et al. The 'dirty dozen' of freshwater science: detecting then reconciling hydrological data biases and errors. WIREs Water e1209, 1-19 (2017).

7. ABI. Data: 'General Insurance Statistics'”'. (2014).

8. Ciurean, R., Gill, J., Reeves, H., O'Grady, S. \& Aldridge, T. Review of environmental multihazards research and risk assessments. 86 (2019). 
9. Palin, E. J. et al. Skillful Seasonal Forecasts of Winter Disruption to the U.K. Transport System. Journal of Applied Meteorology and Climatology 55, 325-344 (2016).

10. Dimitriou, K., McGregor, G. R., Kassomenos, P. A. \& Paschalidou, A. K. Exploring winter mortality variability in five regions of england using back trajectory analysis. Earth Interactions 20, (2016).

11. Wyncoll, D. \& Gouldby, B. Integrating a multivariate extreme value method within a system flood risk analysis. Journal of Flood Risk Management 8, 145-160 (2013).

12. Zscheischler, J. et al. Future climate risk from compound events. Nature Climate Change (2018) doi:10.1038/s41558-018-0156-3.

13. Zscheischler, J. et al. A few extreme events dominate global interannual variability in gross primary production. Env. Res. Lett. 9, 035001 (2014).

14. Kappes, M. S., Keiler, M., von Elverfeldt, K. \& Glade, T. Challenges of analyzing mulithazard risk: a review. Nat. Hazards 64, 1925-1958 (2012).

15. Leonard, M. et al. A compound event framework for understanding extreme events. WIREs Climate Change 5, 113-128 (2014).

16. Walz, M. A., Donat, M. G. \& Leckebusch, G. C. Large-Scale Drivers and Seasonal Predictability of Extreme Wind Speeds Over the North Atlantic and Europe. Journal of Geophysical Research: Atmospheres 123, 11518-11535 (2018).

17. Vecchi, G. A. et al. On the seasonal forecasting of regional tropical cyclone activity. Journal of Climate 27, 7994-8016 (2014).

18. Camargo, S. J., Sobel, A. H., Barnston, A. G. \& Klotzbach, P. J. The influence of natural climate variability on tropical cyclones and seasonal forecasts of tropical cyclone activity. in From Science to Mitigation, World Scientific Series on Asia-Pacific Weather and Climate 325360 (World Scientific, Singapore, 2010). 
19. Patricola, C., Saravanan, R. \& Chang, P. The Impact of the El Nino-Southern Oscillation and Atlantic Meridional Mode on Seasonal Atlantic Tropical Cyclone Activity. Journal of Climate 27, 5311-5328 (2014).

20. Matthews, T., Murphy, C., Wilby, R. L. \& Harrigan, S. Stormiest winter on record for Ireland and UK. Nature Climate Change 4, 738-740 (2014).

21. De Luca, P., Hillier, J. K., Wilby, R. L., Quinn, N. W. \& Harrigan, S. Extreme multi-basin flooding linked with extra-tropical cyclones. Env. Res. Lett. 12, 114009 (2017).

22. Khouakhi, A. \& Villarini, G. Contribution of tropical cyclones to rainfall at global scale. Journal of Climate 30, 359-372 (2017).

23. Berghuijs, W. R., Harrigan, S., Molnar, P., Slater, L. \& Kirchner, J. W. The relative importance of different flood-generating mechanisms across Europe. Water Resour. Res. 55, 4582-4593 (2019).

24. Embrechts, P., McNeil, A. \& Straumann, D. Correlation and dependency in risk management: properties and pitfalls. in Risk Management: Value at Risk and beyond 176223 (Cambridge University Press, Cambridge, UK., 1998).

25. Genest, C. \& Favre, A.-C. Everthing you always wanted to know about copula modelling but were afraid to ask. Journal of Hydrologic Engineering 12, 347-368 (2007).

26. Coles, S., Heffernan, J. \& Tawn, J. Dependence measures for extreme value analyses. Extremes 2, 339-365 (1999).

27. Teugels, J. L. \& Vandewalle, B. Statistical Analysis of Catastrophic Events. Lecture notes in Economics and Mathematical Systems 581, 105-117 (2006).

28. Clemente, A. \& Romano, C. A copula-extreme value theory approach for modelling operational risk. in Operational Risk Modelling and Analysis: Theory and Practice 360 (2004).

29. Murtagh, F. \& Legendre, P. Ward's hierarchical agglomerative clustering method: which algorithms implement Ward's criterion? Journal of Classification 31, 274-295 (2014). 
30. Zscheischler, J. \& Seneviratne, S. I. Dependence of drivers affects risks associated with compound events. Science Advances 3, e1700263 (2017).

31. Mitchell-Wallace, K., Jones, M., Hillier, J. K. \& Foote, M. Natural Catastrophe Risk Management and Modelling: A Practitioner's Guide. (Wiley, 2017).

32. Hewitt, K. \& Burton, I. Hazardousness of a place: a regional ecology of damaging events. (Toronto Press, Toronto, 1971).

33. Seneviratne, S. I. et al. Changes in climate extremes and the impacts upon the natural physical environment. in Managing the Risks of Extreme Events and Disasters to Advance Climate Change Adaptation 109-230 (Cambridge University Press, Cambridge, UK., 2012).

34. Gill, M. \& Malamud, B. D. Reviewing and visualizing the interactions of natural hazards. Reviews of Geophysics 52, 680-722 (2014). 